\title{
A motivação para o desenvolvimento do trabalho de músicos de orquestra
}

\author{
Fausto Kothe (UFPR, Curitiba, PR) \\ fausto.viola@gmail.com
}

Clarissa Stefani Teixeira (UFSC, Florianópolis, SC)

clastefani@gmail.com

Érico Felden Pereira (UFPR, Curitiba, PR)

ericofelden@gmail.com

\section{Eugenio Andrés Díaz Merino (UFSC, Florianópolis, SC)}

merino@deps.ufsc.br

Resumo: A motivação pode interferir na profissão musical, principalmente, pela necessidade constante da prática instrumental. No entanto, a literatura especializada não apresenta com clareza os principais fatores motivacionais para esta atividade profissional. Assim, buscou-se identificar os fatores relacionados à motivação para o desenvolvimento do trabalho de 22 instrumentistas de uma orquestra semi-profissional da região Sul do Brasil. A motivação foi avaliada por meio do questionário de FERREIRA et al. (2006) que apresenta 28 questões que buscam avaliar a motivação para o desenvolvimento das atividades profissionais relacionadas com a organização do trabalho, com realização e poder, com o desempenho e motivação associada ao envolvimento. Para a análise dos dados foi realizada a estatística descritiva por meio de média e desvio padrão e análise fatorial. Os resultados demonstraram que os fatores com a maior pontuação estão associados à motivação para a realização profissional e ao poder dentro da orquestra e com a motivação para o desenvolvimento das atividades profissionais relacionadas com a organização do trabalho. Essas informações se refletem nos resultados da análise fatorial que também indicou ambos os domínios como maior poder de explicação para se ter motivação para o desenvolvimento da prática junto ao instrumento. De forma geral, pode-se dizer que os instrumentistas encontram-se motivados para a realização do trabalho.

Palavras-chave: ergonomia, músicos, orquestra, motivação, trabalho.

\section{The motivation for developing the work of orchestra musicians}

Abstract: Motivation can interfere with the music profession, especially by the constant need for instrumental practice. However, the literature does not show clearly the main motivating factors for this professional activity. Thus, we sought to identify the factors related to motivation for developing the work of 22 musicians from an unprofessional orchestra in southern Brazil. The motivation was assessed by questionnaire FERREIRA et al. (2006) with 28 questions that seek to assess the motivation for the development of professional activities related to the organization of work, achievement and power, with the performance and motivation associated with involvement. For the data analysis was performed descriptive statistics through average and standard deviation and factor analysis. The results showed that the factors with the highest score are associated with motivation for professional achievement and power within the orchestra and the motivation for the development of professional activities related to the organization of work. These information reflect themselves in the results of factor analysis and also indicated that both domains as bigger explanatory power to the motivation for the development of practice with the instrument. In general, one can say that the musicians are motivated to carry out the work.

Keywords: ergonomics, musicians, orchestra, motivation, work. 


\section{1 - Introdução}

Os processos que envolvem as atividades diárias das organizações que buscam a eficácia requerem um comportamento participativo e motivado por parte dos trabalhadores. A motivação é um estado psicológico no qual o indivíduo tem disposição para realizar uma ação, seja no trabalho, seja em qualquer esfera de sua vida (FUCCI-AMATO, 2008). É considerada uma das dimensões mais referenciadas nos vários contextos de intervenção dos psicólogos (REGO, 2002) e, para as intervenções em ergonomia, estratégias que viabilizem alterar a percepção dos trabalhadores podem ser pensadas e aplicadas para a melhora das condições de trabalho.

Segundo FERREIRA et al. (2006) as empresas procuram encontrar argumentos que rentabilizem a produtividade e o desenvolvimento organizacional de forma a identificar nos seus recursos humanos aqueles que desenvolvem maiores índices de motivação e, consequentemente, deem respostas mais eficazes face aos indicadores de performance organizacional.

Especificamente na prática profissional, a motivação é um importante fator não apenas quando se relaciona à produtividade, mas, também, quando se associam as questões de saúde (MARTINEZ e PARAGUAY, 2003). Além disso, as práticas em ergonomia centram suas análises no ser humano, o que possibilita um equilibrio entre a produção e o bem-estar. Segundo FERREIRA et al. (2006), o estudo da motivação no trabalho considera as condições de trabalho responsáveis pelos objetivos, pela qualidade e pela intensidade do comportamento no trabalho.

Em músicos, as investigações que relacionem a motivação mostram-se como uma lacuna de conhecimento, tendo em vista a escassez de estudos relacionados ao tema. FRAGELLI e GÜNTHER (2009) indicam que tal lacuna pode estar relacionada à falsa ideia de que a música se associa apenas ao lazer estando, portanto, raramente relacionada a uma atividade laboral. Essas considerações se refletem nos prejuízos à profissão no que tange as questões de melhora das condições de trabalho e de como proceder para o desenvolvimento da atividade.
Além disso, o estudo da motivação de músicos instrumentistas é importante visto que pode interferir na profissão musical pela necessidade constante da prática junto ao instrumento, que se caracteriza como individual e coletiva. Logo, este estudo buscou identificar os fatores relacionados à motivação para o desenvolvimento do trabalho de instrumentistas de orquestra.

\section{2 - Métodos}

0 estudo foi realizado com uma orquestra semi-profissional da Região Sul do Brasil que foi selecionada por meio de sorteio. A orquestra selecionada possuía em seu quadro de trabalhadores 29 instrumentistas. Para efeito de análise foram investigados os 22 instrumentistas que aceitaram participar do estudo (sendo 15 cordas e sete das madeiras e metais) e assinaram um termo de consentimento livre e esclarecido para inclusão no estudo, conforme Resolução n. ${ }^{\circ}$ 196/96 do Conselho Nacional de Saúde, respeitando os princípios éticos contidos na declaração de Helsinque, além do atendimento à legislação vigente.

A motivação foi avaliada por meio de um questionário construido e validado por FERREIRA et al. (2006). 0 questionário é constituído de 28 itens que avaliam quatro dimensões referentes à motivação no trabalho. Os itens 1, 5, 9, 13, 17, 21 e 25 medem a motivação tendo em vista a organização do trabalho, os itens 2, $6,10,14,18,22$ e 26 estão associados à motivação para o desempenho. As questões 3, 7, 11, 15, 19, 23 e 27 avaliam dimensões referentes aos motivos de realização e poder associados ao trabalho. Por último, os itens 4, 8, 12, 16, 20, 24 e 28, reportamse a aspectos de motivação ligados ao envolvimento com o trabalho. Todas estas afirmações encontramse associadas a uma escala de resposta do tipo likert com cinco tipos de respostas alternativas, cujos posicionamentos de resposta oscilam entre os pólos, 1 - discordo totalmente, 2 - discordo, 3 - não discordo nem concordo, 4 - concordo e 5 - concordo totalmente. A partir da média de cada dimensão são encontrados os escores que indicam a motivação dos indivíduos. Quanto mais próximo de cinco, maior é a motivação relacionada às atividades de trabalho.

\begin{tabular}{lcc}
\hline Componentes do questionário & média & desvio padrão \\
\hline $\begin{array}{l}\text { Motivação para o desenvolvimento das atividades profissionais relacionadas com a } \\
\text { organização do trabalho }\end{array}$ & 3,63 & 0,53 \\
Motivação com o desempenho & 3,62 & 0,31 \\
Motivação com a realização e o poder & 3,71 & 0,64 \\
Motivação associada ao envolvimento & 3,47 & 0,40 \\
\hline
\end{tabular}

Ex.1 - Componentes do questionário de motivação. 
Para a análise dos dados foi utilizada a análise fatorial para componentes principais, com o objetivo de formar agrupamentos relacionados com as 28 questões do questionário da motivação considerando fatores com autovalores maiores que 1 . Com o objetivo de minimizar a relação entre os fatores, foi utilizada a rotação ortogonal "varimax normalized" no cálculo dos fatores. Nestes fatores, foram consideradas significativas aquelas questões que apresentaram o módulo de $p>0,5$. 0 Gráfico "Scree Plot" foi utilizado para visualizar os fatores mais representativos e apresenta a tendência de explicação dos fatores.

\section{3 - Resultados}

Os resultados relacionados aos componentes do questionário de motivação, conforme respostas dos músicos, estão ilustrados no Ex.1.

0 Ex.2 (Scree Plot) apresenta a tendência de explicação dos fatores, na qual é possivel verificar que a análise de componentes principais extraiu nove fatores com autovalores maiores que 1. Esses fatores foram responsáveis por $25,35 \%$ da variância total explicada. Foram consideradas significativas aquelas questões que apresentaram o módulo de $p>0,7$. As questões agrupadas e seus respectivos domínios estão apresentados no Ex.3.

\section{4 - Discussão}

Este estudo buscou identificar os fatores relacionados à motivação para o desenvolvimento do trabalho de instrumentistas de orquestra.

A importância de investigar a motivação relacionada às atividades profissionais é centrada na identificação da persistência e do direcionamento da atenção e do desenvolvimento das atividades realizadas (ARAÚJO e PICKLER, 2008). CUSTODERO (2006) e ADDESSI e PACHET (2007) afirmam que existe uma relação clara entre a persistência no estudo com o instrumento e a satisfação da experiência vivenciada, o que justifica a necessidade de práticas constantes com o instrumento musical.

De forma geral, pode-se dizer que os instrumentistas avaliados no presente estudo encontram-se motivados para o desenvolvimento das atividades laborais, visto que os escores aproximam-se de cinco. Além disso, os resultados demonstraram que os fatores com a maior pontuação estão associados à realização e ao poder dentro da orquestra e com a motivação para o desenvolvimento das atividades profissionais relacionadas com a organização do trabalho (Ex.1). Essas informações se refletem nos resultados da análise fatorial (Ex.2) que também indicou ambos os domínios como maior poder de explicação para se ter motivação para o desenvolvimento da prática junto ao instrumento. Além disso, o estudo de FERREIRA et al. (2006) também encontrou resultados semelhantes. Os autores verificaram $22.22 \%$ de explicação para a motivação face à organização e $8.17 \%$ para a realização e poder.

COSTA (2005) relaciona a organização do trabalho e as diretrizes que trazem à tona questões como realização e poder, de competição, de reconhecimento entre pares, de controle de pressões sofridas. Neste sentido, segundo CUNHA e MARQUES (1990) a motivação no trabalho encontra-se associada à produtividade organizacional, o que corrobora com os resultados encontrados no presente estudo. Dentro desse fator, as questões de maior pontuação com o resultado final estão associadas ao ambiente de cooperação entre colegas de trabalho.

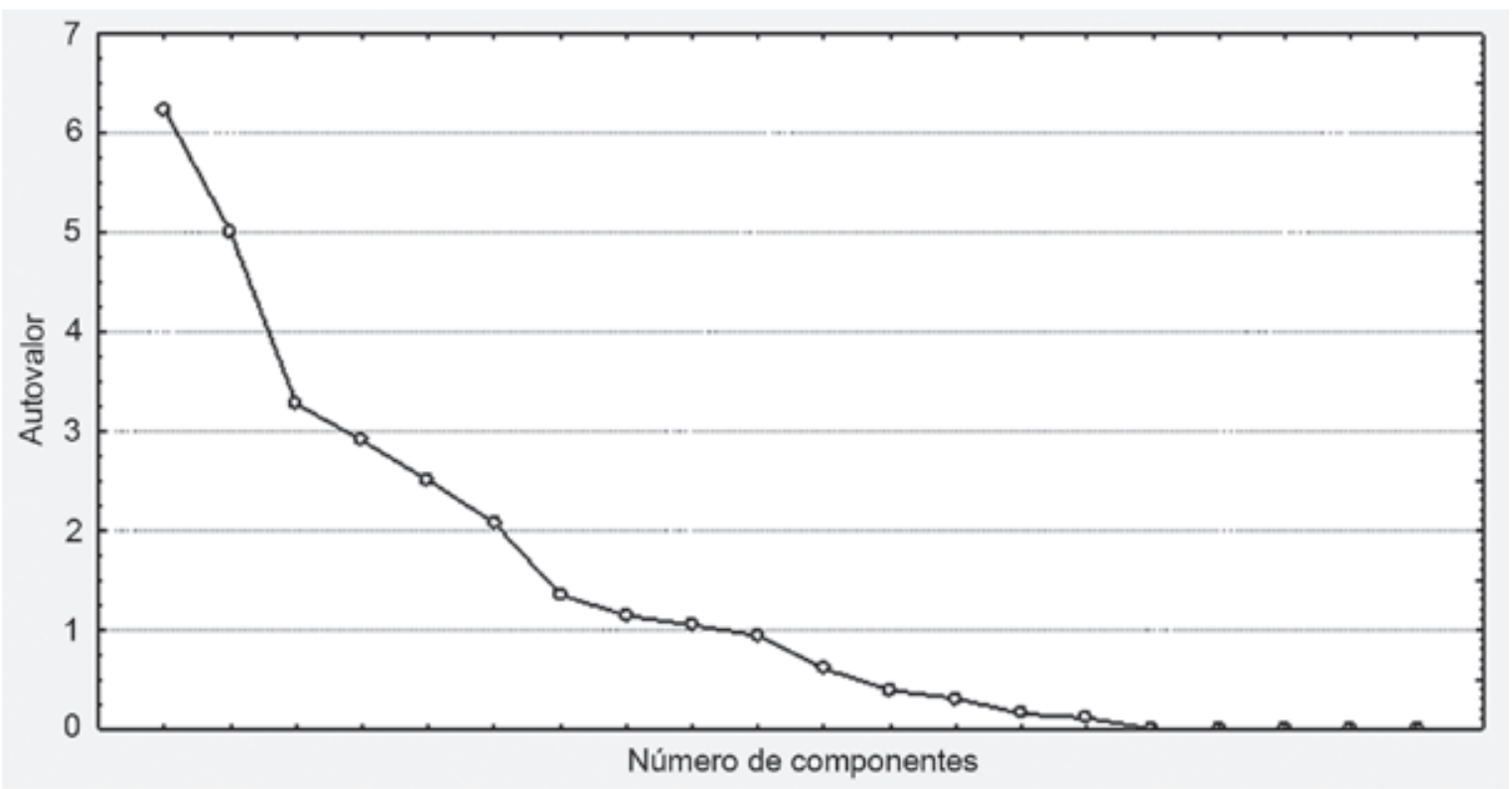

Ex.2 - Tendência de explicação dos fatores da motivação (Scree plot). 


\begin{tabular}{|c|c|c|c|c|}
\hline Fatores & Questões & $\begin{array}{l}\text { Domínios de } \\
\text { Motivação }\end{array}$ & $P$ & $\begin{array}{l}\text { Explicaçãc } \\
(\%)\end{array}$ \\
\hline 1 & $\begin{array}{l}\text { Ter perspectivas de carreira é importante para a minha } \\
\text { motivação no trabalho }\end{array}$ & $\begin{array}{l}\text { realização e o } \\
\text { poder }\end{array}$ & 0,7974 & \multirow{5}{*}{4.33} \\
\hline 1 & $\begin{array}{l}\text { Gostaria de desempenhar funções com maior } \\
\text { responsabilidade }\end{array}$ & $\begin{array}{l}\text { realização e } \\
\text { poder }\end{array}$ & 0,7637 & \\
\hline 1 & $\begin{array}{l}\text { Sinto necessidade de crescer cada vez mais na minha } \\
\text { função }\end{array}$ & $\begin{array}{l}\text { realização e } \\
\text { poder }\end{array}$ & 0,9233 & \\
\hline 1 & $\begin{array}{l}\text { Se existissem prêmios atribuídos aos melhores, percebia- } \\
\text { os como um fator de motivação profissional }\end{array}$ & $\begin{array}{l}\text { realização e } \\
\text { poder }\end{array}$ & 0,8853 & \\
\hline 1 & $\begin{array}{l}\text { Sinto-me motivado(a) quando o trabalho é elogiado pelo } \\
\text { meu superior }\end{array}$ & $\begin{array}{l}\text { realização e } \\
\text { poder }\end{array}$ & 0,7271 & \\
\hline 2 & $\begin{array}{l}0 \text { "feedback" que recebo no trabalho contribui enquanto } \\
\text { fator motivacional }\end{array}$ & $\begin{array}{l}\text { organização do } \\
\text { trabalho }\end{array}$ & 0,7297 & \multirow{3}{*}{4.07} \\
\hline 2 & $\begin{array}{l}\text { Considero que trabalho num ambiente de cooperação } \\
\text { entre colegas }\end{array}$ & $\begin{array}{l}\text { organização do } \\
\text { trabalho }\end{array}$ & 0,9015 & \\
\hline 2 & $\begin{array}{l}\text { A organização permite o desenvolvimento dos objetivos } \\
\text { profissionais }\end{array}$ & $\begin{array}{l}\text { organização do } \\
\text { trabalho }\end{array}$ & 0,7608 & \\
\hline 3 & Identifico-me com a função que desempenho & $\begin{array}{l}\text { envolvimento } \\
\text { com o trabalho }\end{array}$ & 0,6950 & 3.23 \\
\hline 4 & $\begin{array}{l}\text { Sinto-me realizado com as funções que desempenho na } \\
\text { organização }\end{array}$ & $\begin{array}{l}\text { organização do } \\
\text { trabalho }\end{array}$ & 0,9087 & 3.06 \\
\hline 5 & $\begin{array}{l}\text { Todos os indivíduos da organização participam nos } \\
\text { processos de tomada de decisão }\end{array}$ & $\begin{array}{l}\text { organização do } \\
\text { trabalho }\end{array}$ & 0,7011 & 1.89 \\
\hline 6 & Existe competitividade no meu grupo de trabalho. & desempenho & 0,8884 & 2.98 \\
\hline 7 & $\begin{array}{l}\text { Trabalharia com maior empenho se existissem formas de } \\
\text { remuneração alternativa. }\end{array}$ & $\begin{array}{l}\text { envolvimento } \\
\text { com o trabalho }\end{array}$ & 0,8764 & 1.91 \\
\hline 8 & Considero que as avaliações periódicas me motivam & desempenho & 0,7338 & \multirow[b]{2}{*}{2.04} \\
\hline 8 & Considero o meu trabalho monótono. & $\begin{array}{l}\text { envolvimento } \\
\text { com o trabalho }\end{array}$ & 0,7540 & \\
\hline 9 & Sinto-me satisfeito com a minha remuneração & $\begin{array}{l}\text { organização do } \\
\text { trabalho }\end{array}$ & 0,8720 & 1.84 \\
\hline
\end{tabular}

Ex.3 - Distribuição das questões da avaliação da motivação de acordo com os fatores de agrupamento.

Para COSTA e ABRAHÃO (2004) a dimensão coletiva presente na atividade musical exige um ajuste fino dos mecanismos perceptivos, das previsões dos movimentos necessários à produção do som e da intensidade com que este será realizado, das combinaç̧ões e divisões de tarefas na execução com os outros instrumentistas, de forma a manter a música acontecendo.

Além disso, MENDES e ABRAHÃO (1996) encontraram que as restrições e o incentivo ao individualismo dos pares dificultam a construção de um coletivo de trabalho e pode conduzir a um sentimento de impotência frente à estrutura organizacional. $\mathrm{Na}$ música, estas questões são fundamentais visto que há necessidade não só de trabalho individual, mas também de uma interação coletiva para a composição da obra musical. Muitas vezes, as práticas dentro das orquestras são condicionadas pelo rigor/rigidez (JOURDAIN, 1997; GATES, 2001; COSTA, 2005), o que faz com que o trabalho não seja equilibrado, descaracterizando o real papel que cada um tem dentro do todo. Consequentemente, conflitos individuais ou do próprio coletivo podem ser gerados refletindo em sobrecarga cognitiva e física com reflexos negativos no resultado final. 
Assim, segundo MENDES e ABRAHÃO (1996), na medida em que as relações com os pares e hierarquia são fragilizadas pela organização do trabalho, há maior dificuldade em estabelecer o coletivo de trabalho, podendo levar ao sofrimento. Por outro lado, a valorização e o reconhecimento de competências pelos colegas e hierarquia podem ser fontes de vivências de prazer que se aliam aos investimentos sublimatórios propiciados pela atividade, o que, consequentemente, reflete na motivação para o desenvolvimento das atividades. Outra questão que se mostrou importante na análise foi relacionada ao desenvolvimento dos objetivos profissionais que, quando alcançado, se reflete em maior motivação para a continuidade das atividades. MCCLELLAND (1987) indica também que o feedback relacionado ao desempenho durante as atividades é valorizado no que se refere à motivação dos trabalhadores, o que reflete nos resultados encontrados no presente estudo.

\section{MCCORNICK e MCPHERSON (2003) e MCCORNICK e} MCPHERSON (2006) indicam que as práticas atuam como uma parte vital no desenvolvimento da capacidade de realização instrumental e esta, por sua vez, deve considerar as variáveis motivacionais. Com relação à pontuação da motivação relacionada à realização e ao poder, parece que os elogios dos superiores durante a prática instrumental e o recebimento de prêmios pelas atividades desenvolvidas seriam importantes fatores. Para ARAÚJO e PICKLER (2008) o papel do professor que auxilia o desenvolvimento das atividades musicais é fundamental no que tange a motivação da aprendizagem. Neste sentido, LOCKE e LATHAM (1990) referem que avaliações positivas melhoram a eficácia do trabalho e reforçam a motivação.

A profissão musical determina ainda a necessidade do desempenho de funções com maior responsabilidade. 0 que ocorre é que a responsabilidade dos músicos é apenas relacionada à execução de seu próprio instrumento musical, sendo as demais atividades da orquestra de responsabilidade do maestro/regente ou outros membros integrantes da orquestra. Estas considerações indicam a necessidade de crescimento relatada pelos músicos. Vale lembrar que dentro do naipe de instrumento existe essa possibilidade o que, consequentemente, faz com que novos estudos sejam realizados junto ao instrumento para se conseguir o posto de spala da orquestra, ou chefe de naipe do instrumento.

Para TAMAYO e PASCHOAL (2003) a motivação laboral é uma das áreas em pesquisa e gestão de recursos humanos que tem sido sempre orientada por uma abordagem positiva e humanizadora do ambiente de trabalho, procurando encontrar caminhos para ajudar o trabalhador a satisfazer as suas necessidades no trabalho e a se realizar profissionalmente nele. Assim, relativamente à necessidade de realização, esta pode definir-se como um impulso de realização em direção a um conjunto de padrões, em que se insere o desejo de querer ser excelente e de ser bem sucedido em situações de competição (FERREIRA et al., 2006). Estas considerações vão ao encontro da presença de perspectivas da carreira apresentada pelos instrumentistas avaliados.

Essas questões se refletem nos componentes que mostraram menores motivações. Estes, segundo o Ex.1, estão associados com a designação de envolvimento com as atividades do trabalho $(3,47 \pm 0,40$ pontos $)$ e podem, mais uma vez estar associados à limitação das atividades relacionadas com seu próprio instrumento de trabalho. Enquanto existe a possibilidade de sobrecarga física quando se desenvolve atividades repetitivas e de precisão como os desenvolvidos com os instrumentos de orquestra, segundo SKINNER (1989) a variedade de funções, identidade e significado das tarefas refletem a forma como o trabalho se encontra estruturado e dependem dos conhecimentos associados ao trabalho e afetam a motivação.

Além disso, a realização de tarefas que impossibilitem variedade pode tornar 0 trabalho monótono $e_{\text {, }}$ consequentemente, diminuir a motivação para o desenvolvimento do mesmo. Para TAMAYO e PASCHOAL (2003) o problema da motivação no trabalho situa-se, inevitavelmente, no contexto da interação dos interesses da organização com os interesses do empregado. As duas partes envolvem-se numa parceria, na qual cada uma delas apresenta e explicita suas exigências e demandas.. Para FERREIRA et al. (2006) as caracteristicas estão relacionadas com a importância atribuida às tarefas e estas contribuem para uma maior satisfação no trabalho através da motivação intrínseca. Por outro lado, quando o trabalho permite alguma autonomia, os empregados relacionam a performance com os seus esforços e decisões, na medida em que o trabalhador sente o crescimento da sua responsabilidade pelo trabalho.

Outra preocupação associada aos baixos valores de motivação para o domínio do envolvimento com as atividades realizadas é a indicação de ALLEN e MEYER (1997) que afirmam que o envolvimento com as atividades é uma medida determinante na produtividade e que parece estar associada enquanto elemento que contribui fortemente para a motivação no trabalho.

A remuneração recebida na orquestra avaliada também parece não motivar o desenvolvimento das atividades junto à orquestra. Neste sentido, TAMAYO e PASCHOAL (2003) relacionam que a valorização dos empregados está associada à satisfação dos trabalhadores. Para CARVALHO e SILVA (2006) em uma era de competitividade a remuneração fixa tornou-se insuficiente para motivar e incentivar as pessoas a obter um comportamento proativo, empreendedor e eficaz na busca de metas e resultados excelentes. Para os autores, diferentemente dos resultados encontrados, a remuneração não apresenta associação com a motivação, pois funciona apenas como fator higiênico. 
As maiores problemáticas em se ter trabalhadores desmotivados podem estar associadas às indicações de EREZ (1997), que define a interferência na disposição para dedicar esforço, conhecimentos e habilidades pessoais no seu trabalho. Assim, a valorização dos trabalhadores é de fundamental importância se a organização pretender manter um lugar de destaque no mercado altamente competitivo de hoje.

\section{5 - Conclusão}

Os resultados demonstraram que os fatores com a maior pontuação estão associados à motivação com a realização e com o poder dentro da orquestra $(3,71 \pm 0,64$ pontos) e com a motivação para o desenvolvimento das atividades profissionais relacionadas com a organização do trabalho $(3,63 \pm 0,53$ pontos). Essas informações se refletem nos resultados da análise fatorial que também indicou ambos os domínios como maior poder de explicação para se ter motivação para o desenvolvimento da prática junto ao instrumento. De forma geral, pode-se dizer que os instrumentistas encontram-se motivados para a realização do trabalho.

Porém, ter perspectivas na carreira mostrou-se importante para a motivação. Deste modo, estratégias como promoções e planos de carreira, facilitações para a realização de cursos superiores e outros cursos poderiam ser estratégias dentro da orquestra, o que demonstraria melhorias não apenas para a qualificação dos trabalhadores como também para a motivação.

Conforme indicam FERREIRA et al (2006), as influências no trabalho são multifatoriais, o que leva a uma necessidade de novos estudos no tocante da ergonomia. Assim, recomenda-se que medidas de promoção da saúde e trabalho dos músicos, intervenções ergonômicas bem como políticas públicas no contexto do trabalho considerem os fatores que mais motivam os músicos para sua atuação profissional, buscando resultados mais eficientes.

\section{Referencias}

ADDESSI, A. R.; PACHET, F. Sistemas musicais interativos-reflexivos para educação musical. Cognição e Artes Musicais, v. 2, n.1, p.62-72, 2007.

ALLEN, N. J.; MEYER, J. P. Affective, continuance, and normative commitments to the organization: An examination of construct validity. Journal of Vocational Behaviour, v. 49, p.252-276, 1990.

ARAÚJO, R. C.; PICKLER, L. Um estudo sobre a motivação e o estado de fluxo na execução musical. In: IV SIMPÓSIO DE COGNIÇÃO E ARTES MUSICAIS, 4, Curitiba, 2008. Anais... Curitiba: Universidade Federal do Paraná, 2008.

CARVALHO, A. C.; SILVA, B. V. M. A motivação para o trabalho com enfoque no aspecto financeiro. In: XIII SIMPÓSIO DE ENGENHARIA DA PRODUÇÃO, 13, Bauru, 2006. Anais... Bauru: Universidade Estadual Paulista, 2006.

COSTA, C. P. Contribuições da ergonomia à saúde do músico: considerações sobre a dimensão física do fazer musical. Música Hodie, v. 5, n. 2, p.53-63, 2005.

COSTA, C. P.; ABRAHÃO, J. I. Quando o tocar dói: um olhar ergonômico sobre o fazer musical. Performance Musical, v. 10, p.60-79, 2004.

CUNHA, M. P.; MARQUES, C. A. Relação entre Objectivos/Feedback e Desempenho/Envolvimento/ Satisfação. Análise Psicológica, v. 8, n. 3, p.295-304, 1990.

CUSTODERO, L A. Buscando desafios, encontrando habilidades: a experiência de fluxo e a educação musical. In: ILARI, Beatriz (Org.). Em busca da mente musical. Curitiba: Editora da UFPR, 2006, p.381-399.

EREZ, M. A culture-based model of work motivation. In: EARLY, C.; EREZ, M. (Eds.). New perspectives on international industrial/organizational psychology. San Francisco: The New Lexington Press, 1997. p.193-242.

FERREIRA, A.; DIOGO, C.; FERREIRA, M.; VALENTE, A. C. Construção e validação de uma Escala Multi-Factorial de Motivação no Trabalho (Multi-Moti). Comportamento Organizacional e Gestão, v. 12, n. 2, p.187-198, 2006.

FRAGELLI, T. B.; GÜNTHER, I. A. Relação entre dor e antecedentes de adoecimento físico ocupacional: um estudo entre músicos instrumentistas. Performance Musical, n. 19, p.18-23, 2009.

FUCCI AMATO, R. C. 0 canto coral como prática sócio-cultural e educativo-musical. Opus, v. 13, n. 1, p.75-96, 2007.

GATES, D. The Philharmonic's new admiral takes command. Newsweek, v. 138, n. 2, p.56-57, 2001.

JOURDAIN, R. Música, cérebro e êxtase. Rio de Janeiro: Objetiva, 1997.

LOCKE, E. A.; LATHAM, G. P. A theory of goal setting and task performance. Englewood Cliffs, NJ: Prentice-Hall, 1990.

MARTINEZ, M. C.; PARAGUAY, A. I. B. B. Satisfação e saúde no trabalho: aspectos conceituais e metodológicos. Cadernos de Psicologia Social do Trabalho, v. 6, p.59-78, 2003. 
MCCLELLAND, D. C. Human Motivation. Cambridge: Cambridge University Press, 1987.

MCCORNICK, J.; MCPHERSON, G. E. The role of self-efficiancy in a musical performance examination: an exploratory structural equation. Psychology of Music, v. 31, n. 1, p.37-51, 2003.

MCCORNICK, J.; MCPHERSON, G. E. Self-efficacy and performing music. Psychology of Music, v. 3, n. 3, p.321-336, 2006.

MENDES, A. M.; ABRAHÃO, J. I. Organização do trabalho e vivências de prazer-sofrimento do trabalhador: abordagem psicodinâmica. Psicologia: Teoria e Pesquisa, v. 12, p.179-184, 1996.

REGO, A. Os motivos de sucesso, afiliação e poder: desenvolvimento e validação de um instrumento de medida. Análise Psicológica, v. 18, n. 3, p.335-344, 2000.

SKINNER, B. F. Recent issues in the analysis of behaviour. Columbus: Merrill Publishing Company, 1989.

TAMAYO, A.; PASCHOAL, T. A relação da motivação para o trabalho com as metas do trabalhador. Revista de Administração Contemporânea, v. 7, n. 4, p.33-54, 2003.

Fausto Kothe: Possui graduação em Música (bacharelado em viola) pela Universidade Federal de Santa Maria (2004). Apresentou-se com as principais orquestras do estado do Rio Grande do Sul, Santa Catarina e Paraná. Atuou na docência e preparação de alunos para a graduação durante os anos de 2006-2009. Atualmente é mestrando em Música da Universidade Federal do Paraná na linha de pesquisa Educação Musical, Cognição e Filosofia.

Clarissa Stefani Teixeira: Possui graduação em Educação Física pela Universidade Federal de Santa Maria (2004). Realizou especialização em Atividade Física, Desempenho Motor e Saúde na linha de pesquisa Biomecânica da Atividade Física pela Universidade Federal de Santa Maria (2005). É Doutora em Distúrbios da Comunicação Humana pela Universidade Federal de Santa Maria (2006-2008). Atualmente tem seus estudos voltados para a saúde e qualidade de vida dos trabalhadores atuando principalmente na área de ergonomia, antropometria e biomecânica. É doutoranda em Engenharia de Produção da Universidade Federal de Santa Catarina na linha de pesquisa de Ergonomia e é bolsista CNPq.

Érico Felden Pereira: Possui graduação em Educação Física (2004) e especialização em Atividade Física, Desempenho Motor e Saúde (2006) pela Universidade Federal de Santa Maria. É mestre em Educação Física pela Universidade Federal de Santa Catarina (2008) e doutor em Educação Física pela Universidade Federal do Paraná. Tem experiência nas áreas de Saúde e Educação, atuando principalmente nos seguintes temas: barreiras e facilitadores para a promoção da qualidade de vida das pessoas e comunidades, saúde escolar, desigualdades em saúde, comportamento motor, sono, sonolência diurna excessiva, educação física escolar e imagem corporal. Atualmente é professor efetivo da Universidade do Estado de Santa Catarina.

Eugenio Andrés Díaz Merino: Possui graduação em Desenho Industrial pela Universidade Federal do Rio de Janeiro (1993), mestrado em Engenharia de Produção pela Universidade Federal de Santa Catarina (1996) e doutorado em Engenharia de Produção pela Universidade Federal de Santa Catarina (2000). Atualmente é professor adjunto IV da Universidade Federal de Santa Catarina e coordena o Núcleo de Gestão de Design. Atualmente atua nos seguintes temas: ergonomia, gestão de design e usabilidade. Participa dos programas de Pós-Graduação em Design e Engenharia de Produção da UFSC. Faz parte do grupo de avaliadores do INEP/MEC e do Conselho Estadual de Educação de Santa Catarina na avaliação de cursos. 